www.jmscr.igmpublication.org

Impact Factor (SJIF): 6.379

Index Copernicus Value: 71.58

ISSN (e)-2347-176x ISSN (p) 2455-0450

crossref DOI: https://dx.doi.org/10.18535/jmscr/v6i4.41

Journal Of Medical Science And Clinical Research

\title{
Ultrasound grading of fatty liver and association with obesity
}

\author{
Authors \\ Muhammad Abdullah, AM Anand, P Elamparidhi, R Ramesh Kumar \\ Corresponding Author \\ Muhammad Abdullah \\ SMVMCH \\ Email: meetthesurgeon@gmail.com
}

\begin{abstract}
Introduction: The most common incidental finding in an ultrasound abdomen scan is fatty liver. The significance of this finding is a curiosity for the radiologist and the clinician as there has been lots of study with equivocal results.

Aim: To study if there is any significant relationship between grades of fatty liver and hepatomegaly with mild, moderate or morbid obesity.

Materials and Methods: This study was done as a hospital based retrospective study in department of Radiology for a duration of one month in all patients who were incidentally detected with fatty liver. BMI was calculated for correlation with hepatomegaly and grading of fatty liver.
\end{abstract}

\section{Introduction}

Fatty liver is the most commonly detected incidental finding in ultrasound. It is seen in patients of all age groups and affects both males and females. Obesity is a major health problem worldwide. In the United States, roughly 300,000 deaths per year are related to obesity ${ }^{1 .}$

Obesity is associated with an increased risk of nonalcoholic fatty liver disease (NAFLD). Steatosis, the hallmark feature of NAFLD, occurs when the rate of hepatic fatty acid uptake from plasma and de novo fatty acid synthesis is greater than the rate of fatty acid oxidation and export (as triglyceride within VLDL). Therefore, an excessive amount of intrahepatic triglyceride represents an imbalance between complex interactions of metabolic events ${ }^{2}$.
The results from one study, conducted in a cohort of Hispanic and non-Hispanic Caucasians and African American subjects, who were considered to be at low-risk for NAFLD (i.e. BMI $<25 \mathrm{~kg} / \mathrm{m} 2$, no diabetes, and normal fasting serum glucose and alanine aminotransferase concentrations), suggest the threshold for a normal amount of IHTG should be $5.6 \%$ of liver volume, because this value represented the 95th percentile for this "normal" population ${ }^{3}$.

The prevalence rate of NAFLD increases with increasing body mass index (BMI). An analysis of liver histology obtained from liver donors, automobile crash victims, autopsy findings, and clinical liver biopsies suggests that the prevalence rates of steatosis and steatohepatitis are approximately $15 \%$ and $3 \%$, respectively, in nonobese persons, $65 \%$ and $20 \%$, respectively, in 
persons with class I and II obesity (BMI 30.0$39.9 \mathrm{~kg} / \mathrm{m} 2$ ), and $85 \%$ and $40 \%$, respectively, in extremely obese patients (BMI $\geq 40 \mathrm{~kg} / \mathrm{m} 2$ ). The relationship between BMI and NAFLD is influenced by racial/ethnic background and genetic variation in specific genes ${ }^{4}$.

Optimum sensitivity for liver US was achieved at a liver fat content $\geq 12.5 \%$, suggesting that below this threshold, liver US is less sensitive. Liver $1 \mathrm{H}-$ MRS showed a high accuracy for the diagnosis of NAFLD, and correlated strongly with histological steatosis $(r=0.73, \mathrm{P}<0.0001)^{5}$.

\section{Materials and Methods:}

This study is was done as a hospital based Cross sectional study department of Radiology for a duration of one month in all patients who are incidentally detected with fatty liver. BMI was calculated for correlation with hepatomegaly and grading of fatty liver.

\section{Results}

\section{Sex distribution}

Out of the 40 patients, there was slight predilection for fatty liver among male patients being $52 \%$. In grade $\mathrm{I}$, there was slight predilection for male patients $(52 \%)$ compared to females. In grade II, both males and females show equal distribution, In grade III, There was slight male predominance $(66 \%)$.

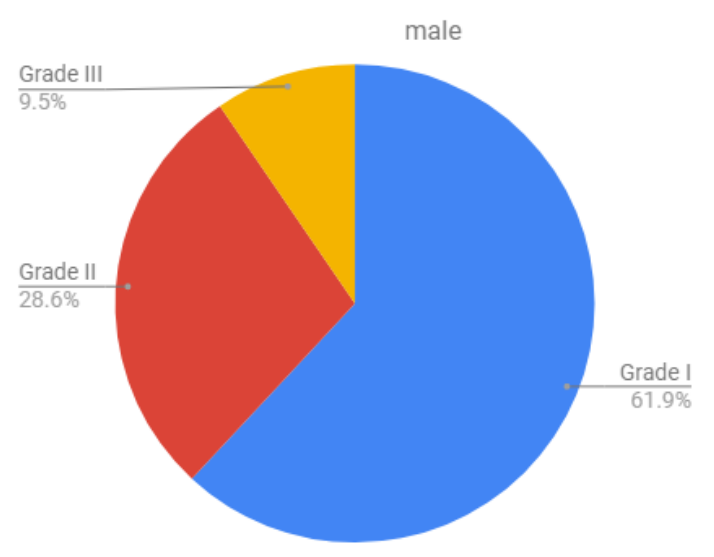

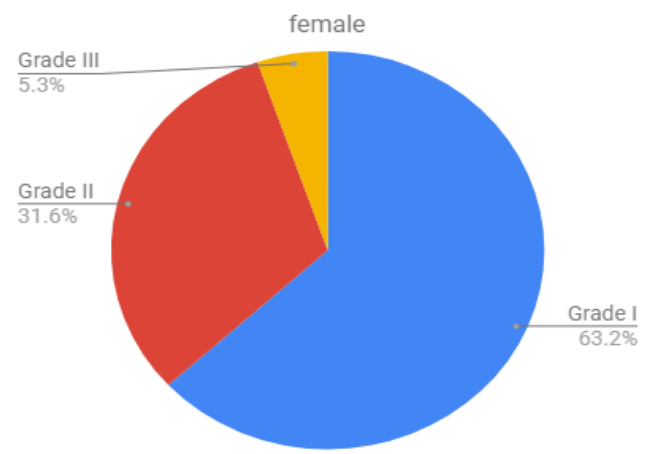

\section{Age distribution}

The mean age of presentation was 40 years, The distribution of fatty liver regardless of grading was $2.5 \%$ in age range of $0-20,45 \%$ in the age range of $20-40,47 \%$ in the age range of $40-60$ and $5 \%$ in the age range of $60-80$.

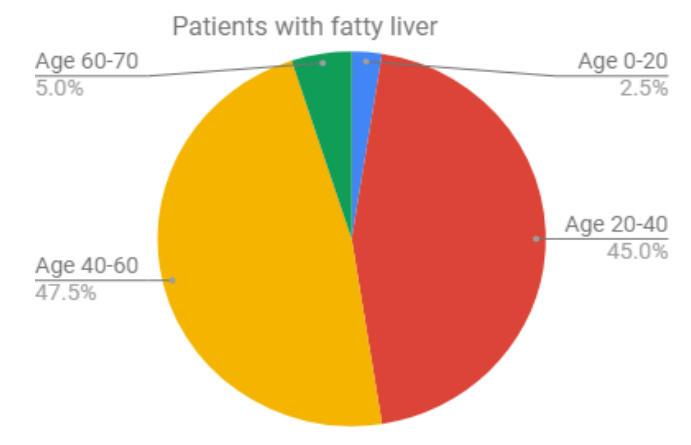

\section{Fatty liver in relationship with weight}

The majority of patients with fatty liver in this study were between the weight $60-80 \mathrm{~kg}$ comprising $57 \%$ of the cases. Out of which $56 \%$ had grade I fatty liver; 39\% had grade II fatty liver and $4 \%$ had grade I fatty liver.

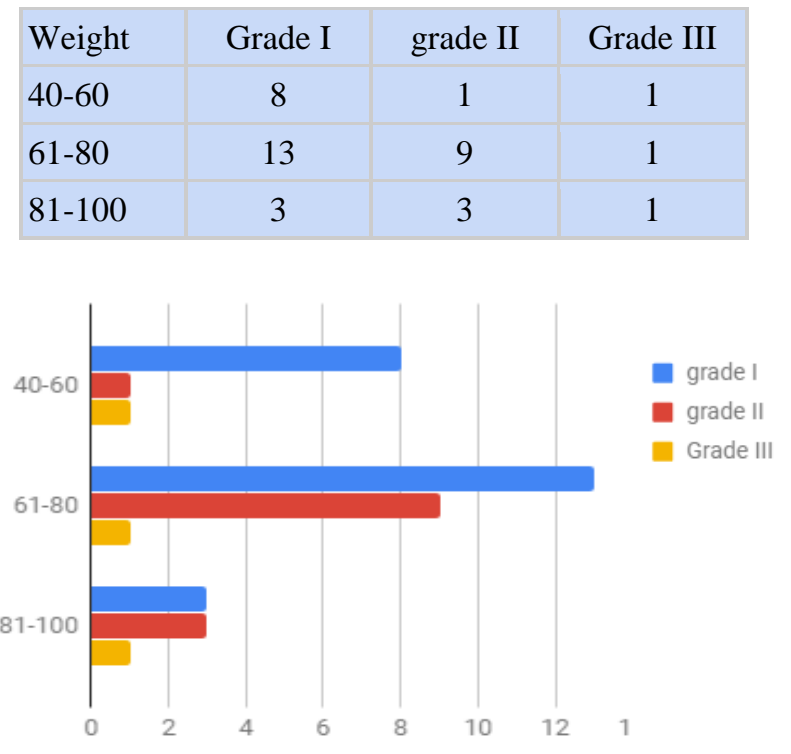




\section{JMSCR Vol||06||Issue||04||Page 244-248||April}

Relationship of fatty liver with hepatomegaly

Patients with Grade I fatty liver predominantly had their liver size less than $15 \mathrm{~cm}$ in $76 \%$ of cases, $20 \%$ between $15-17 \mathrm{~cm}$ and less than $4 \%$ more than $17 \mathrm{~cm}$.

Those who had grade II fatty liver had liver sizes less than $15 \mathrm{~cm}$ in $58 \%$ of cases; $15-17 \mathrm{~cm}$ in $25 \%$ of cases and more than $17 \mathrm{~cm}$ in $16.7 \%$ of cases.
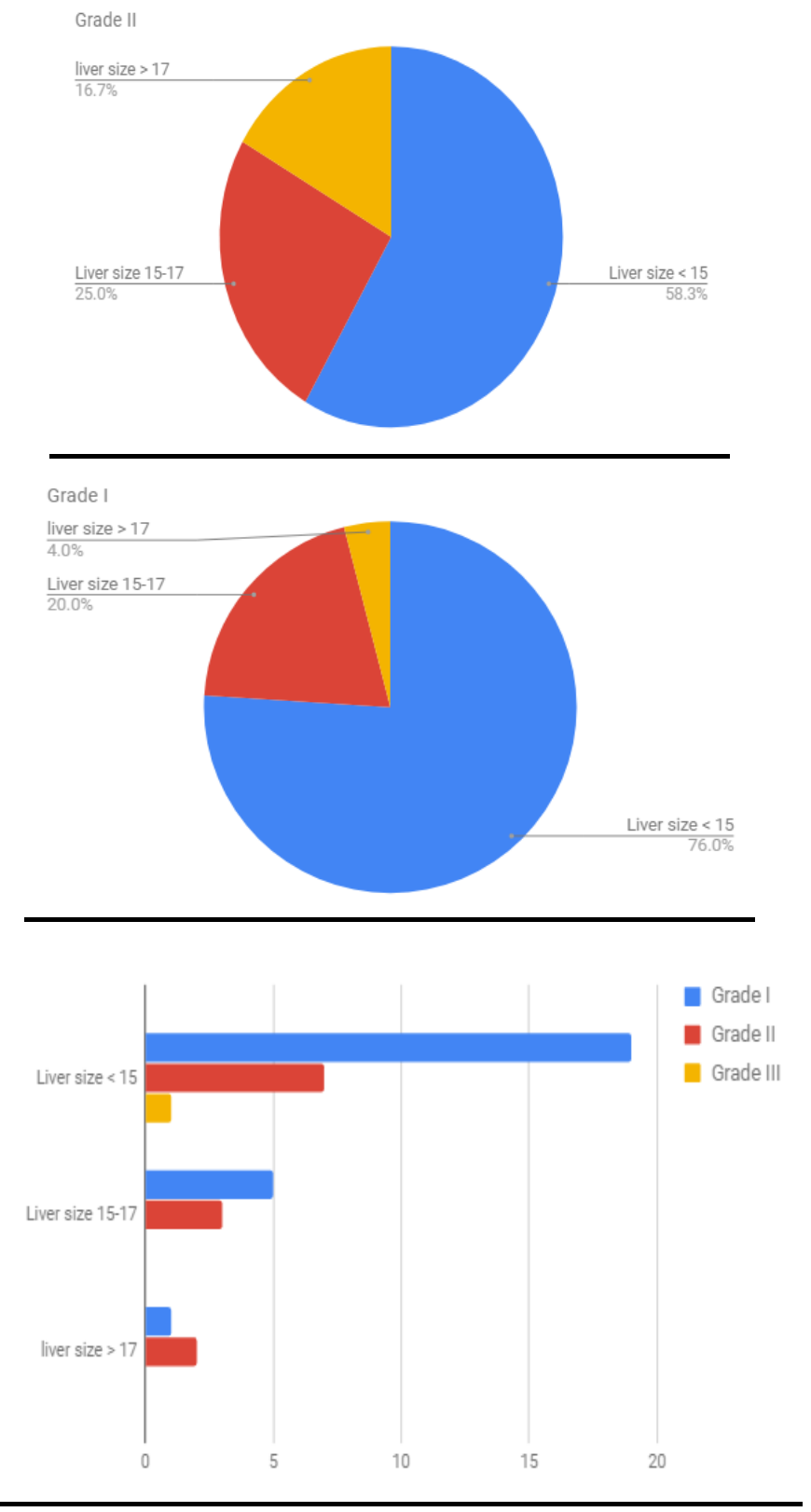

Relationship of BMI with fatty liver and hepatomegaly

Among the study cases, the mean BMI was 27.5. $70 \%$ of patients had BMI more than 25. Among these $85 \%$ patients had BMI between 25 and 35 .

\begin{tabular}{|l|c|c|c|}
\hline & BMI 14-25 & BMI 26-35 & BMI 36-45 \\
\hline Grade I FL & 8 & 15 & 1 \\
\hline Grade II FL & 2 & 7 & 2 \\
\hline Grade III FL & 1 & 2 & 0 \\
\hline
\end{tabular}

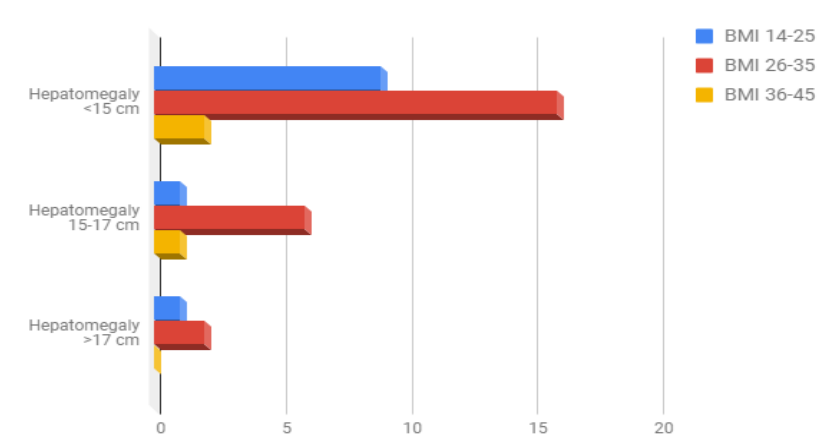

Among the patients with fatty liver, $67 \%$ of patients did not have hepatomegaly, 20\% cases had mild to moderate hepatomegaly and $7.5 \%$ had gross hepatomegaly. $66 \%$ of grade III fatty liver had no significant hepatomegaly.

\begin{tabular}{|l|c|c|c|}
\hline & BMI 14-25 & BMI 26-35 & BMI 36-45 \\
\hline Hepatomegaly $<15 \mathrm{~cm}$ & 9 & 16 & 2 \\
\hline Hepatomegaly $15-17 \mathrm{~cm}$ & 1 & 6 & 1 \\
\hline Hepatomegaly $>17 \mathrm{~cm}$ & 1 & 2 & 0 \\
\hline
\end{tabular}
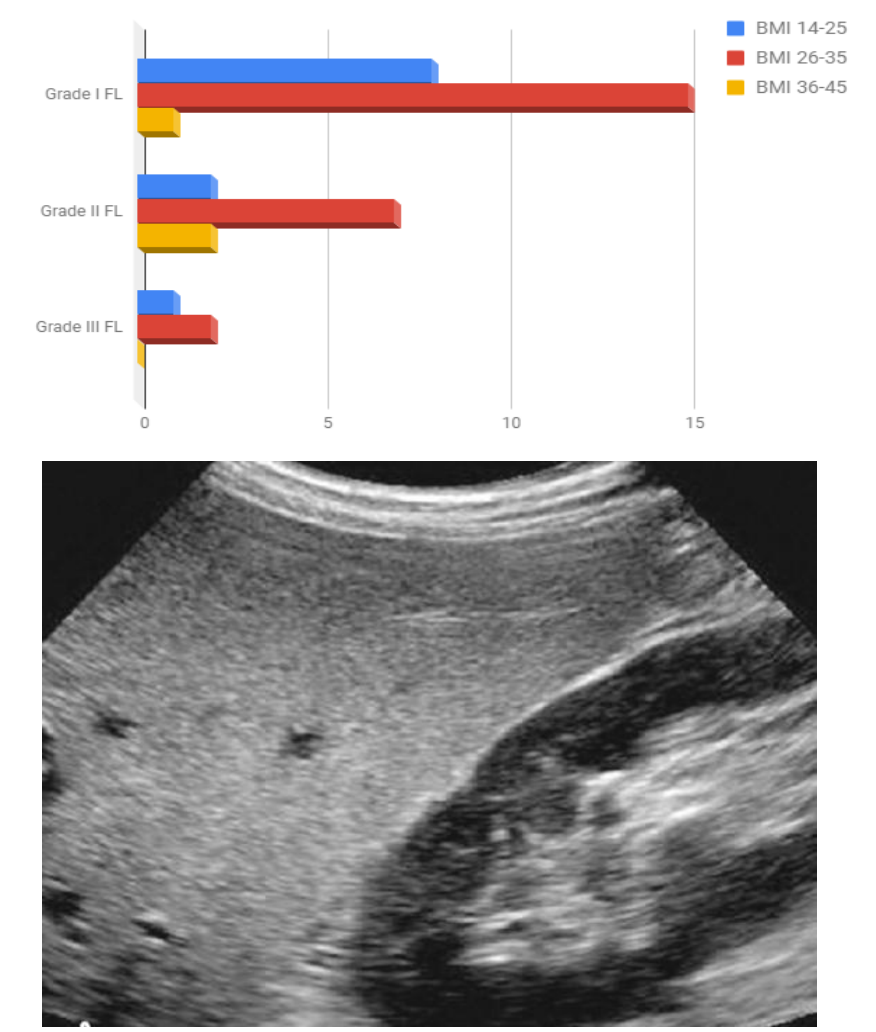

Image of grade I fatty liver, The liver appears hyperechoic than the kidney. 


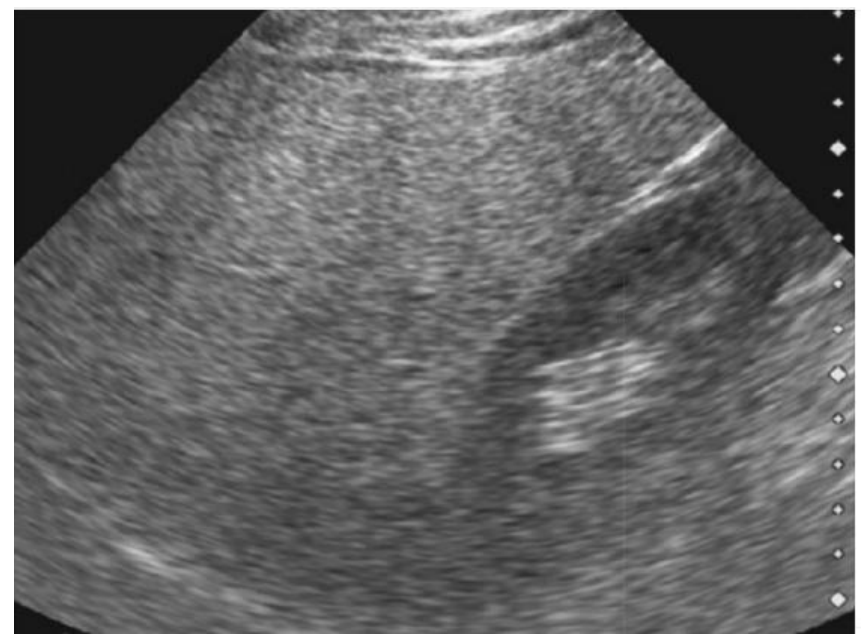

Image of grade II fatty liver, The diaphragm is faintly seen.

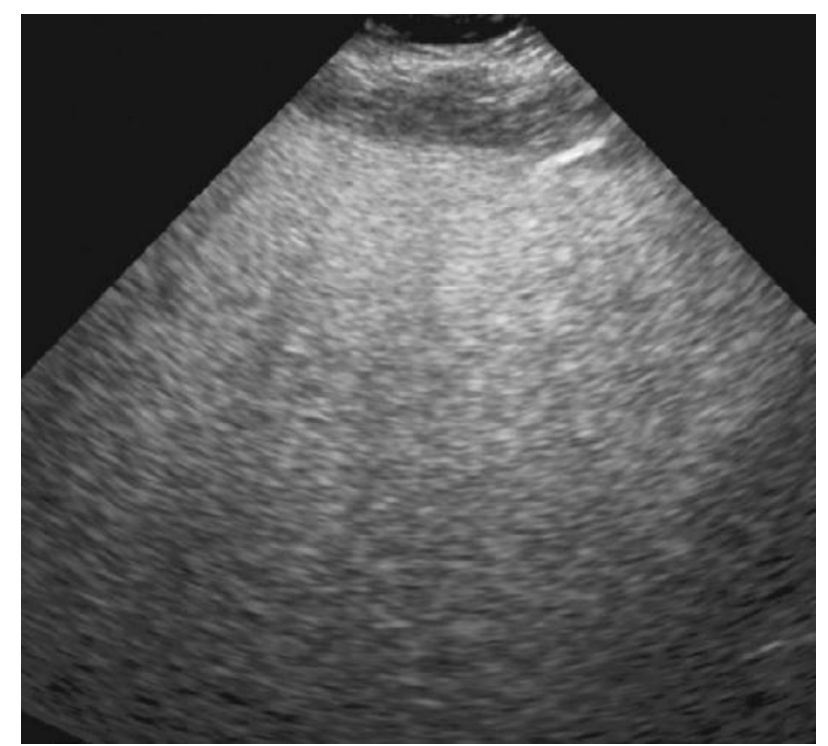

Image of grade III fatty liver. The diaphragm is not seen at all

\section{Discussion}

The majority of patients affected with fatty liver are between the age of 40 and 60 . There is no significant male or female predominance in case of fatty liver. The mean age affecting patients with fatty liver is around 40 years.

The maximum number of cases of fatty liver (70\%) were seen to have a BMI between 26-35 suggesting that there is significant correlation between moderately obese patients and fatty liver irrespective of the grade (as one patient with BMI less than 25 had grade III fatty liver with normal liver size in this study.)

There is a significant correlation between obesity and fatty liver as $70 \%$ of cases with BMI more than 25 were seen to have been affected with fatty liver in our study; however there is no significant correlation between hepatomegaly and fatty liver. The maximum number of patients affected weigh between 60 and 80 kilograms.

Interestingly, $25 \%$ of cases in our study had fatty liver whose BMI were below 25 - suggesting that obesity is not the only factor contributing to fatty liver. Other associated/ environmental/ social factors may be the cause in these patients.

\section{Conclusion and clinical implication}

Incidentally detected fatty liver in moderately obese patients show correlation with BMI, however, obesity is not the only reason for fatty liver. Fatty liver detection in young patients and with normal BMI patients may help the clinician evaluate a patient for hyperlipidemia and prevent complications of it if present

The mere presence of fatty infiltration show some correlation with obesity but grading of fatty liver does not show any correlation between mild/ moderate/ severe obesity.

Severe fatty infiltration maybe seen in non-obese patients, while mild fatty infiltration maybe seen in severely obese patients; implying that the severity of infiltration multifactorial.

Patients with incidentally detected fatty liver should be evaluated further on two grounds.

1. Biochemical evaluation.

2. Lifestyle evaluation.

It is advisable for any patient with fatty liver to undergo basic biochemical investigation such as the lipid profile and blood sugars to rule out metabolic syndrome or familial hypercholesterolemia.

Other possible lifestyle factors should also be asked for such as sedentary lifestyle, alcohol consumption, high fatty diet. Suggestions has to be made on how to improve their habits.

Persisting fatty liver despite normal biochemical and adequate lifestyle changes does not require treatment or further follow up unless nonalcoholic steatohepatitis is ruled out. 


\section{References}

1. Toshikuni N. Clinical differences between alcoholic liver disease and nonalcoholic fatty liver disease. World Journal of Gastroenterology. 2014;20(26):8393.

2. Fabbrini E, Sullivan S, Klein S. Obesity and nonalcoholic fatty liver disease: Biochemical, metabolic, and clinical implications. Hepatology. 2009;51(2):679689.

3. Shaye K, Amir T, Shlomo S, Yechezkel S. Fasting glucose levels within the high normal range predict cardiovascular outcome. American Heart Journal. 2012;164(1):111-116.

4. Rinella M. Body mass index as a predictor of hepatic steatosis in living liver donors. Liver Transplantation. 2001;7(5):409-414.

5. Bril F, Ortiz-Lopez C, Lomonaco R, Orsak $\mathrm{B}$, Freckleton $\mathrm{M}$, Chintapalli $\mathrm{K}$ et al. Clinical value of liver ultrasound for the diagnosis of nonalcoholic fatty liver disease in overweight and obese patients. Liver International. 2015;35(9):21392146. 